\title{
The Deactivation Network in Brain During Acute Stress
}

\author{
Shigang Feng ${ }^{1}$, Wei Wang ${ }^{2}$, Hongbo Liu ${ }^{1,3}$, Ajith Abraham ${ }^{3,4}$ \\ ${ }^{1}$ School of Information, Dalian Maritime University, Dalian 116026, China \\ ${ }^{2}$ Physical Science and Technical College, Dalian University, Dalian 116622, China \\ ${ }^{3}$ Machine Intelligence Research Labs, WA 98071, USA \\ ${ }^{4}$ VSB Technical University of Ostrava, Czech Republic \\ fsg1512@163.com,wangwei-612@163.com, lhb@dlut.edu.cn, ajith.abraham@ieee.org
}

\begin{abstract}
There is growing evidence from patient and psychoneuroimmunology studies that there is a clear correlation between the psychosocial stress and health state, but the neural mechanism of the correlation remain poorly understood. The deactivations of the limbic system and anterior frontal cortex during acute psychosocial stress were reported in recent studies, and the observed results suggest these brain areas are activated at rest and during nonstressful situations suggesting the deactivation regions during stress play an important role in detrimental health effects of psychosocial stress. However, these studies mostly aim at confining the brain regions that present the decrease of the brain blood flow. The response mode and space-time relation of these regions from overall and dynamic angle are left untouched. In this paper, we exposed human subjects to one mathematic stressor and one comparison task in one functional magnetic resonance imaging (fMRI; $n=17$ ) experiment and one of the regions of interest (ROIs)-based functional connectivity analyses, within-condition interregional covariance analysis (WICA), is employed to analyze the interaction of the brain regions that show deactivation during different task states. We demonstrate a profound deactivation of limbic system and anterior frontal cortex during mathematic stress and a neural network of deactivation regions, which was modulated by the difficult degree of mathematic tasks.
\end{abstract}

Keywords-fMRI; Deactivation; Mental arithmetic stress; functional connectivity: WICA;

\section{INTRODUCTION}

There is growing evidence from patient and neuroimaging studies that there is a clear correlation between the psychosocial stress and health state. If the stress-response is activated chronically, it can be the cause of a variety of disorders such as myopathy, fatigue, hypertension, ulceration, psychogenic dwarfism, amenorrhea, impotency, increased disease risk and neuron death [1], [2], [3]. However, neural activation patterns associated with the initiation of the stress response remain poorly understood. In recently researches, The deactivations of the limbic system and anterior frontal cortex during acute psychosocial stress were reported [4], and the observed results suggest these brain areas are activated at rest and during nonstressful situations suggesting the activation regions play an important role in detrimental health effects of psychosocial stress [5], [6], [7]. However, these studies mostly aim at confining the brain regions that present the decrease of the brain blood flow. The response mode and space-time relation of these regions from overall and dynamic angle are left untouched.

Some of the past studies have provided evidence that the limbic system and anterior frontal cortex play an important role in the self regulation [8], [9]. So we can assumed that stress may cause deactivations of brain areas associated with self regulation, involving the prefrontal cortex, hippocamplus, insula, precune and anterior cingulate cortex [4]. In this study, we exposed human subjects to one mathematic stressor and one comparison task in one functional magnetic resonance imaging (fMRI; $n=17$ ) experiment and one of the ROI-based functional connectivity analyses, withincondition interregional covariance analysis (WICA), is employed to analyze the interaction of the brain regions that show deactivation during different task states.

\section{MATERIALS AND METHODS}

\section{A. Subjects}

A total of 17 healthy young subjects (10 men, mean age $23.35 \pm 3.17$ years; 7 women, mean age $22.75 \pm 3.11$ years) recruited from the local population of graduates or undergraduates at Dalian University of Technology participated in this study. Informed consent was obtained before participation.

\section{B. Behavioral Tasks}

Psychological stress was induced using the Mental Arithmetic Task (MAT), where subjects are exposed to challenging mental arithmetic presented on a computer screen, to which they have to respond using a tow-button mouse in time. In each trial of the experimental condition, initially there appear two Arabic numbers with "+" or "-" between them, after 0.5 second, the third number appeared. For the experimental condition, subjects had to decide whether or not the third number was larger than the operation results of the first two in 1 second. After each trial, feedbacks were presented to subjects. If subjects did not respond correctly and in good time, they would receive the negative feedbacks using sad face picture as well as raspy sound. In the control condition, white circular or triangular graphics 
were presented as visual stimuli in the triplets presented without feedbacks after each trial. The sequence of events for the Control task was the same as that in the mental arithmetic tasks. The task was to decide whether or not the third graphics in a triplet had the same shape as the pair of graphics presented first. In the rest condition, the user interface is displayed with a small white attention dot and without deciding tasks being shown.

\section{Functional Imaging Data Acquisition and Analysis}

Subjects were scanned on a 3.0 T Siemens Magnetom Vision Scanner, employing a block design with two runs. In two runs, three experimental or control blocks were presented in a constant order with three rest blocks being presented alternately, each rest block was preceding each experimental or control block, see Fig.1).

All fMRI data were analyzed by using SPM8 software packages (Wellcome Department of Cognitive Neurology, Institute of Neurology, London). Images were first spatially realigned, normalized, and smoothed $(8 \mathrm{~mm}$ full width at half-maximum). Statistical analysis used a random effect model, with individual activation maps being generated using the general linear model. The voxel-wise threshold for activation was set at $P<0.05$, corrected for the number of resolution elements in each of the ROIs by using the SPM small volume correction (SVC) procedure together with brain masks defined by the automated anatomical labeling toolbox ${ }^{1}$. The brain masks defined the brain regions over each of which the SVC was performed. These brain regions included the prefrontal cortex, hippocamplus, insula, precune and anterior cingulate cortex, which were used for further functional connectivity analysis. Differences in the task-induced deactivation between the two conditions were examined with two sample $t$ tests, and statistical threshold was set at $P<0.001$ uncorrected.

We applied the within-condition interregional covariance analysis (WICA) method for ROI-based functional connectivity analyses [10], [11]. For each task, functional modulation of brain circuitry was modeled through cross-correlation analysis of blood oxygen level-dependent (BOLD) signal over three task blocks and over 17 subjects. In short, in the current study, we limited our analysis in the preselected ROIs or a network of areas that were deactivated during the experimental and control condition (Fig. 1). These areas included the mPFC (medial prefrontal cortex), the ACC (anterior cingulated cortex), the insular, and the hippocampus in the left hemisphere (Fig. 2). The averaged BOLD signal for each task block was individually calculated for each ROI in a network defined both anatomically and functionally on the activation maps. The between-ROI correlation was then calculated for the experimental and control conditions (Fig. $3)$, and the significance level $(P<0.05)$ of the correlation

\footnotetext{
${ }^{1}$ http://www.fil.ion.ucl.ac.uk/spm/software/spm8/
}

between activation magnitudes was assessed separately by using one-tailed t tests.

\section{RESULT}

\section{A. Brain Deactivations in Mathematic Stress}

In this research, we observed deactivations in a network of structures linked to the limbic system including anterior cingulate cortex, posterior cingulated cortex, left hippocampus, left insular and bilaterally medial frontal cortex. More over, we also observed significant deactivations bilaterally in temporal gyrus and precuneus. Although the similar deactivated networks were found in two conditions, all regions deactivations are significantly larger in experimental condition than those in control condition. See Fig. 2, the results are visualized using $x j$ View toolbox ${ }^{2}$, threshold at uncorrected $P<0.005$.

\section{B. Brain functional connectivity of deactivation regions}

To investigate the interaction of the brain regions that show deactivation during different task states, we used an fMRI connectivity analysis method [10], [11], which yielded distinct functional pathways corresponding to the experimental and control tasks, respectively. Our results show that the activity in right medial frontal gyrus were strongly correlated with the activity in right medial frontal gyrus and activity in anterior cingulate cortex for both experimental and control tasks; the activity in anterior cingulate cortex were strongly correlated with the activity in left medial frontal gyrus, left insular, left hippocampus, right Precuneus and posterior cingulated cortex only for experimental task, and there was strong functional connectivity between left insular and left hippocampus and left amygdala had strong negative correlation to left hippocampus, anterior cingulate cortex and posterior cingulated cortex only for experimental task. See Fig.3.

\section{Discussion}

This present study was to investigate the effects of mathematic stress on brain deactivations with fMRI. Acute and chronic stressors like emotional or physical trauma, or prolonged psychosocial stress are often investigated for their potential implication in health and disease. While the effects of stress on memory and cognition are frequently investigated [12], [13], [14], neural activation patterns associated with the stress response remain poorly understood. Recently, Lupien et al. [12] used a mental arithmetic task in a functional fMRI environment. Authors reported deactivations of limbic system components, arguing that the degree of deactivation in the hippocampus was correlated with the release of cortisol in response to the stress task, and the observed deactivation of limbic system structures suggests elevated activation during nonstressful situations [4]. In this

\footnotetext{
${ }^{2}$ http://www.alivelearn.net/xjview
} 


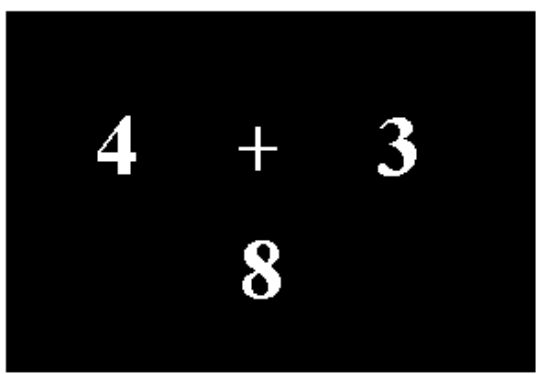

The experimental condition

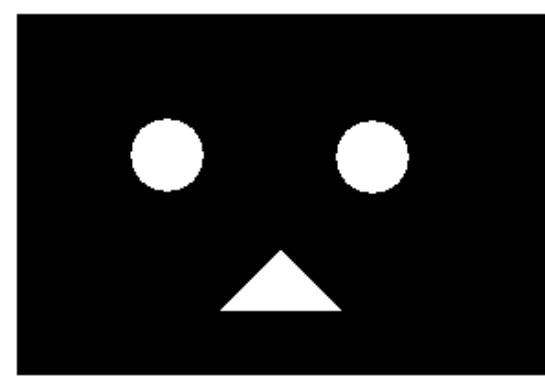

The control condition

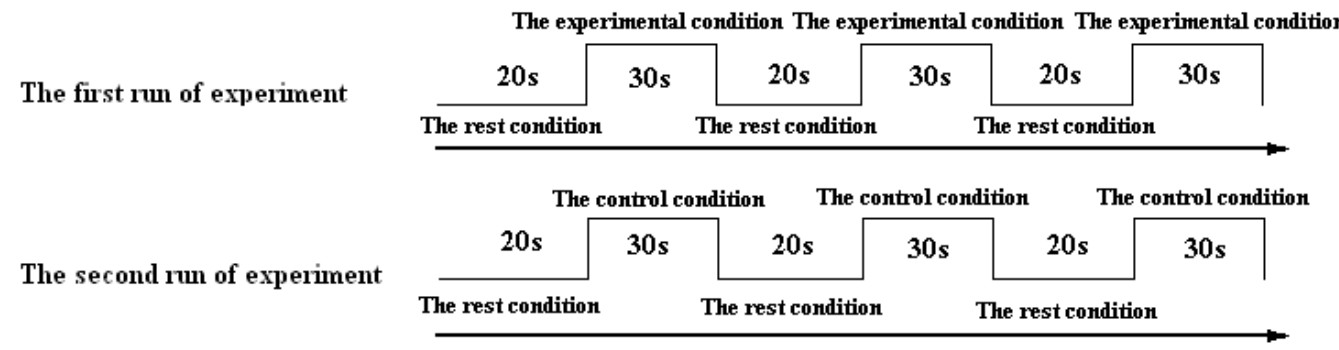

Figure 1. Exemplars of the stimuli used for the tasks and experimental design. A block design was used. Each task condition lasted for $30 s$, each rest condition lasted for $20 \mathrm{~s}$.

study, similar results were found about deactivation of brain areas. In consideration of the functions in which these areas participate [4], we can suggest that the anterior cingulate cortex, the insula, the hippocampus, poster cingulate cortex, precuneus, and the medial frontal cortex are active areas during the resting state or nonstressful situations and play important roles in self-regulation after stress reaction. We can conclude that the deactivation amounts of brain areas during acute stress are positive correlated to the difficult degrees of stressors.

To further demonstrate the above-mentioned conclusions, we used one of the ROI-based functional connectivity analyses, within-condition interregional covariance analysis (WICA), to analyze the interaction of the brain regions that show deactivation during two conditions, which showed more stronger connectivity between deactivation regions in experimental condition than those in control condition (Fig.3). In view of the above-mentioned conclusions that the deactivation regions we found in this study play important roles in self-regulation after stress reaction, we can arrival at a conclusion. As the stress increases, the brain gradually suppresses the operation of a network which plays important roles in self-regulation after stress reaction, and the inhibition of this network may be the key reason for the impairment to human health by the stress.

Seeing from Fig. 3, the anterior cingulate cortex (ACC) plays an important role in the reaction to stress. In the recent years, more and more evidences support a view that the anterior cingulate is involved in the regulation of autonomic activity according to the current context of behavior and the self-regulation after stressing psychosomatic reaction. In general, self-regulation is a natural function of brain networks, designed to control the influx of information from the environment through orienting, in order to avoid conflicting responses in behavior. Many studies present strong evidences that the anterior cingulate gyrus appears to play a role in a wide variety of autonomic functions, such as regulating blood pressure and heart rate, as well as rational cognitive functions, such as reward anticipation, decisionmaking, empathy and emotion [15], [16]. There is also evidence for anatomical connectivity between the ventral cingulate and limbic areas and the dorsal cingulate, parietal, and frontal areas [17]. Anatomical and electrophysiological studies show there is one type of projection cell which called the Von Economo neuron in the anterior cingulate gyrus and stress can diminish the production of these neurons [18], [19], [20]. Thus it is conceivable that the psychosocial stressor may affect the survival and development of the spindle cells during infancy, thus influencing adult competence or dysfunction in emotional self-control and problem-solving capacity.

\section{CONCLUSION}

In conclusion, our results suggest the deactivations of the limbic system and anterior frontal cortex during stressing situation play an important role in impaired effects to human 


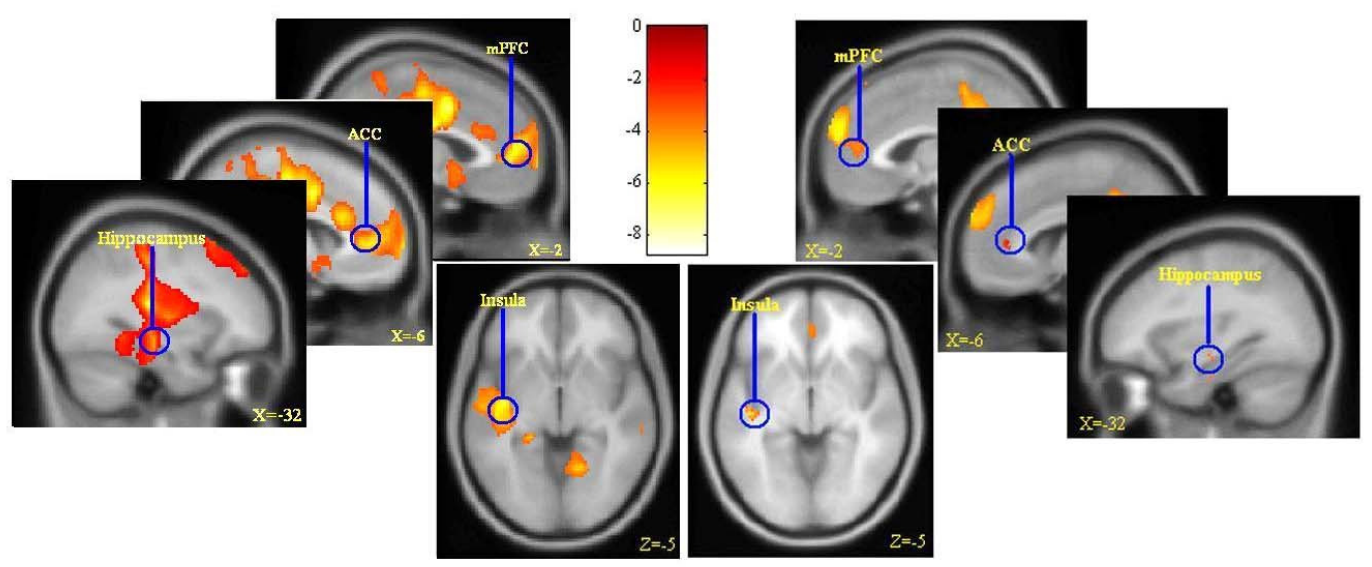

A: Experimental Condition

B: Control Condition

Figure 2. The statistical parametric maps of brain deactivations during processing two conditions relative to baseline.

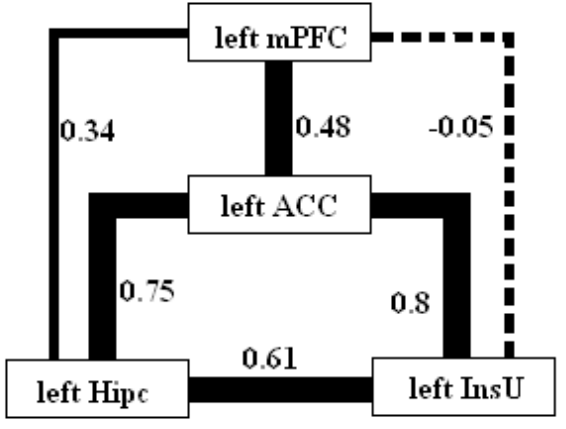

A: Experimental Condition

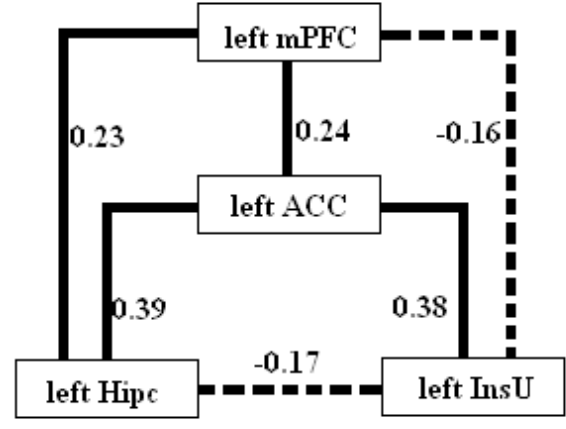

B: Control Condition

positive correlation $(C>0.45)$

positive correlation $(0<C<0.45)$

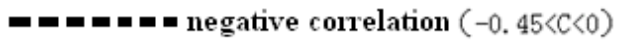

Figure 3. The functional connectivity between ROIs during two conditions. The strength of functional connectivity between ROls was labeled by a number and weighted by lines. mPFC: medial prefrontal cortex; ACC: anterior cingulate cortex; InsU: insular; HipC: hippocampus.

health by psychosocial stress. It is known to all that acute and chronic stressors can impair human health. However, it remains unanswered how the stressors achieve its impaired effects in the neural system. The results of this study provide a hypothesis that the deactivation network of limbic system components and anterior frontal cortex during the presence of psychosocial stress may be the key reason for the impairment to human health by the stress. Future studies should include measures of stress perception, as well as a satisfactory assessment of the amounts to the deactivations in brain to investigate the detailed underlying mechanism.

\section{ACKNOWLEDGMENT}

This work is supported by the National Natural Science Foundation of China (Grant No.60873054, 61073056, 10947174), the Fundamental Research Funds for the Central Universities (Grant No.2011JC006), and Dalian Science and Technology Fund (Grant No.2010J21DW006). Ajith Abraham acknowledges the support of the IT4Innovations Centre of Excellence Project, reg. no. CZ.1.05/1.1.00/02.0070 
supported by Operational Programme 'Research and Development for Innovations' funded by Structural Funds of the European Union and state budget of the Czech Republic.

\section{REFERENCES}

[1] R. Sapolsky, "Why stress is bad for your brain," Science, vol. 273, no. 5276, p. 749, 1996.

[2] — - "Glucocorticoids, stress, and their adverse neurological effects: relevance to aging* 1," Experimental Gerontology, vol. 34, no. 6, pp. 721-732, 1999.

[3] K. Mizoguchi, M. Yuzurihara, A. Ishige, H. Sasaki, D. Chui, and T. Tabira, "Chronic stress differentially regulates glucocorticoid negative feedback response in rats," Psychoneuroendocrinology, vol. 26, no. 5, pp. 443-459, 2001.

[4] J. Pruessner, K. Dedovic, N. Khalili-Mahani, V. Engert, M. Pruessner, C. Buss, R. Renwick, A. Dagher, M. Meaney, and S. Lupien, "Deactivation of the limbic system during acute psychosocial stress: evidence from positron emission tomography and functional magnetic resonance imaging studies," Biological psychiatry, vol. 63, no. 2, pp. 234-240, 2008.

[5] S. Lazar, G. Bush, R. Gollub, G. Fricchione, G. Khalsa, and $\mathrm{H}$. Benson, "Functional brain mapping of the relaxation response and meditation," Neuroreport, vol. 11, no. 7, p. 1581, 2000 .

[6] J. Wang, H. Rao, G. Wetmore, P. Furlan, M. Korczykowski, D. Dinges, and J. Detre, "Perfusion functional mri reveals cerebral blood flow pattern under psychological stress," Proceedings of the National Academy of Sciences of the United States of America, vol. 102, no. 49, p. 17804, 2005.

[7] M. Tillfors, T. Furmark, I. Marteinsdottir, and M. Fredrikson, "Cerebral blood flow during anticipation of public speaking in social phobia: a pet study," Biological Psychiatry, vol. 52, no. 11, pp. 1113-1119, 2002.

[8] A. Newberg, A. Alavi, M. Baime, M. Pourdehnad, J. Santanna, and E. d'Aquili, "The measurement of regional cerebral blood flow during the complex cognitive task of meditation: a preliminary spect study," Psychiatry Research: Neuroimaging, vol. 106, no. 2, pp. 113-122, 2001.

[9] H. Critchley, C. Mathias, O. Josephs, J. ODoherty, S. Zanini, B. Dewar, L. Cipolotti, T. Shallice, and R. Dolan, "Human cingulate cortex and autonomic control: converging neuroimaging and clinical evidence," Brain, vol. 126, no. 10, p. 2139, 2003.

[10] A. He, L. Tan, Y. Tang, G. James, P. Wright, M. Eckert, P. Fox, and Y. Liu, "Modulation of neural connectivity during tongue movement and reading," Human brain mapping, vol. 18 , no. 3, pp. 222-232, 2003.

[11] Y. Tang, W. Zhang, K. Chen, S. Feng, Y. Ji, J. Shen, E. Reiman, and Y. Liu, "Arithmetic processing in the brain shaped by cultures," Proceedings of the National Academy of Sciences, vol. 103, no. 28, p. 10775, 2006.
[12] S. Lupien, C. Wilkinson, S. Brière, C. Ménard, $\mathrm{N}$. Ng Ying Kin, and N. Nair, "The modulatory effects of corticosteroids on cognition: studies in young human populations," Psychoneuroendocrinology, vol. 27, no. 3, pp. 401-416, 2002.

[13] J. McGaugh and B. Roozendaal, "Role of adrenal stress hormones in forming lasting memories in the brain," Current Opinion in Neurobiology, vol. 12, no. 2, pp. 205-210, 2002.

[14] O. Wolf, "Hpa axis and memory," Best Practice \& Research Clinical Endocrinology \& Metabolism, vol. 17, no. 2, pp. 287-299, 2003.

[15] J. Decety and P. Jackson, "The functional architecture of human empathy," Behavioral and Cognitive Neuroscience Reviews, vol. 3, no. 2, p. 71, 2004

[16] P. Jackson, E. Brunet, A. Meltzoff, and J. Decety, "Empathy examined through the neural mechanisms involved in imagining how i feel versus how you feel pain," Neuropsychologia, vol. 44, no. 5, pp. 752-761, 2006.

[17] M. Posner, B. Sheese, Y. Odludas, and Y. Tang, "Analyzing and shaping human attentional networks," Neural Networks, vol. 19, no. 9, pp. 1422-1429, 2006.

[18] J. Allman, K. Watson, N. Tetreault, and A. Hakeem, "Intuition and autism: a possible role for von economo neurons," Trends in Cognitive Sciences, vol. 9, no. 8, pp. 367-373, 2005.

[19] G. Kempermann, H. Kuhn, and F. Gage, "More hippocampal neurons in adult mice living in an enriched environment," vol. 386, pp. 493-495, 1997.

[20] E. Gould, B. McEwen, P. Tanapat, L. Galea, and E. Fuchs, "Neurogenesis in the dentate gyrus of the adult tree shrew is regulated by psychosocial stress and nmda receptor activation," The Journal of neuroscience, vol. 17, no. 7, p. 2492 , 1997. 